\title{
Auto-eficácia, doenças crônicas e incapacidade funcional na velhice
}

\author{
Dóris Firmino Rabelo ${ }^{1}$ \\ Chrystiane Mendonça Cardoso
}

\section{Resumo}

A doença crônica, maior causa de incapacidade entre os idosos, é de grande interesse porque é influenciada por fatores médicos, psicológicos e sociais. O objetivo desta revisão é mostrar a relevância da crença de auto-eficácia como mecanismo protetor ou mediador no ajustamento de idosos incapacitados. Pesquisas indicam que quanto maior as crenças de auto-eficácia, menor a incapacidade, o distress psicológico, os sintomas depressivos, o declínio em atividades básicas e instrumentais da vida diária; e maior a saúde percebida, o ajustamento à dor, o esforço despendido em atividades requeridas, o ajustamento pessoal e a capacidade de mobilização de recursos de enfrentamento.
\end{abstract}

Palavras-chave: Auto-eficácia; Incapacidade funcional; Idosos.

\begin{abstract}
Self-efficacy, cronical diseases and functional disability in the old age
Abstract

Cronical diseases, the major cause of disability among the elderly, are of greater interest because it is influenced by medical, social and psychological factors. The aim of this review is to show the relevance of self-efficacy belief as a protective or mediator mechanism in the adjustment of disable elderly people. Researches show that the higher the self-efficacy beliefs, the lower the disability, the psychological distress, the depressive symptoms, the decrease in daily life basic and instrumental activities; and the higher the perceived health, the pain adjustment, the effort made in required activities, the adjustment and the capacity of personal coping.
\end{abstract}

Keywords: Self-efficacy; Functional incapacity; Elderly.

\section{Introdução}

É comum nas pessoas com idade avançada um quadro de enfermidades complexas e onerosas, o que implica maior utilização de serviços de saúde e maior número de problemas de longa duração. Segundo estudo descritivo de Lima-Costa, Barreto e Giatti (2003) sobre as condições de saúde e o uso de serviços de saúde da população brasileira, 69\% dos idosos relatam ter pelo menos uma doença crônica, sendo a hipertensão e a artrite as mais comuns. A doença crônica é dominante e a maior causa de incapacidade, especialmente entre os mais velhos. Segundo Melzer e Parahyba (2004), a prevalência de incapacidade funcional na população idosa brasileira é maior em mulheres, aumenta com o avançar da idade e a disparidade econômica e educacional são seus indicadores mais importantes.

A saúde e a funcionalidade física na velhice são uma preocupação central no campo do envelhecimento, uma vez que são muitas as dificuldades cotidianas que a incapacidade funcional decorrente de problemas de saúde trazem tanto para os próprios idosos quanto para suas famílias. Incapacidade funcional é comumente definida como a restrição da capacidade do indivíduo de desempenhar atividades normais da vida diária e quantifica o impacto de doenças ou acidentes. Refere-se também a limitações específicas no desempenho de papéis socialmente definidos e de tarefas dentro de um ambiente sociocultural e físico particular. Estão incluídas as atividades básicas e instrumentais de vida diária, os papéis no trabalho, os papéis não-ocupacionais e os papéis recreacionais ou de lazer. A incapacidade exerce grande efeito no bem-estar individual, na necessidade de assistência formal e informal e nos cuidados de longa permanência (Jette, 1996; Lamb, 1996).

As doenças crônicas e as incapacidades conseqüentes podem afetar significativamente o bemestar dos idosos. De acordo com Smith, Borchelt, Maier e Jopp (2002), as condições crônicas levam a uma diminuição do potencial dos indivíduos de experienciar o lado positivo da vida, especialmente entre aqueles acima dos 80 anos de idade. Em estudo com homens de 50 a 72 anos hipertensos, com alto risco de doença cardiovascular, Agewall, Wikstrand, Dahlöf e Fagerberg (1996) encontraram que pacientes com qualquer doença cardiovascular sentem-se significativamente mais

Endereço para correspondência:

${ }^{1}$ Unicamp - Rua Professora Guaraciaba, 246 - Tabajaras - 38400-266 - Uberlândia-MG - E-mail: drisrabelo@yahoo.com.br 2UFU - Rua Tapajós, 110 - Vigilato Pereira - 38400-266 - Uberlândia-MG - E-mail: chryscardoso@prove.ufu.br 
descontentes quando comparados com pacientes sem sinais ou sintomas de doenças cardiovasculares. Segundo Xavier, Ferraz, Marc, Escostequy e Moriguchi (2003), para os idosos octogenários residentes em comunidade da cidade de Veranópolis, a falta de saúde é o principal indicador da ausência de bem-estar e de qualidade de vida negativa.

Entre aqueles afligidos pelas doenças crônicas, alguns avançam para incapacidades severas e outros não. Tem-se pouco entendimento dos fatores que protegem e/ou moderam o impacto das doenças crônicas na vida dos idosos. Para entender porque a pessoa se torna incapacitada é preciso considerar os fatores extraindividuais (como os cuidados médicos, a reabilitação e o ambiente físico e social), os fatores de risco (como as características sociodemográficas e os estilos de vida) e os fatores intra-individuais (como os atributos psicológicos e sociais). Nesse sentido, é levado em conta como o indivíduo avalia a situação da incapacidade e reage a ela, como os outros avaliam a situação da incapacidade e dispõem expectativas para os idosos, e também as características do próprio ambiente físico (Jette, 1996).

O tópico da incapacidade funcional é de grande interesse não apenas porque ela é influenciada por condições médicas, patologia ou deficiência, mas também porque é influenciada por fatores psicológicos e sociais. Os atributos psicológicos podem ajudar a identificar as pessoas com grande risco de perda das capacidades funcionais. Idosos com menos recursos psicológicos estão particularmente em risco de desenvolver incapacidade (Kempen e cols., 1999). Segundo Jang, Haley, Small e Motimer (2002), estudos recentes mostram o papel substancial dos fatores psicossociais no desenvolvimento do processo de incapacidade e sua interação com a mesma. Os fatores psicológicos refletem a percepção subjetiva do indivíduo e sua avaliação da situação, são importantes na adaptação à incapacidade, funcionam como recursos de enfrentamento atenuando a adversidade de situações eestresseantes e auxiliam no manejo do ambiente social e físico.

Os fatores psicossociais têm grande potencial para determinar em que medida a vida estendida será vivida eficazmente ou com debilidade, dor e dependência (Bandura, 2004). Dentre os atributos psicológicos, a crença de auto-eficácia percebida é o foco de interesse deste artigo. Esta revisão tem como objetivo comentar sobre a relevância da crença de auto-eficácia como mecanismo protetor ou mediador no ajustamento pessoal de indivíduos mais velhos com incapacidades. Serão apresentados estudos que indicam como esse recurso psicológico pode operar sobre os efeitos negativos das condições crônicas na vida dos idosos e sobre o enfrentamento das perdas em funções físicas e papéis sociais.

\section{Auto-eficácia no contexto da teoria social cognitiva}

Em 1977, Albert Bandura, representante da teoria social cognitiva, introduziu o conceito de autoeficácia (AE) como sendo a crença que o indivíduo tem na sua capacidade para desempenhar um comportamento específico ou realizar tarefas futuras. Mais tarde, em 1997, o autor ampliou este conceito afirmando que as crenças de $\mathrm{AE}$ são as crenças que as pessoas têm em sua capacidade de organizar e executar cursos de ação necessários para alcançar determinados resultados.

Para Bandura (1997), a expectativa de AE pessoal determina se o comportamento será iniciado, quanto esforço será empreendido e por quanto tempo esse comportamento será mantido diante de obstáculos e experiências aversivas. A força das convicções das pessoas em sua própria efetividade prediz se elas irão tentar enfrentar as situações dadas. Sendo assim, a AE percebida influencia a escolha do ambiente comportamental, ou seja, as pessoas tendem a evitar situações ameaçadoras que elas acreditam exceder suas habilidades de enfrentamento, mas elas se envolvem em atividades e se comportam confiantemente quando se julgam capazes de manusear as situações. A AE não só pode ter influência direta na escolha das atividades e dos ambientes, mas, mediante expectativas de sucessos eventuais, ela pode afetar os esforços de enfrentamento uma vez que foram iniciados. Quanto maior a $\mathrm{AE}$ percebida, mais ativos são os esforços.

As crenças de $\mathrm{AE}$ se originam e se alteram por meio de quatro fontes: experiência de domínio (êxito); experiência vicária; persuasão social e excitação emocional (Bandura 1977, 1997). As experiências de êxito são as mais influentes, já que o próprio indivíduo experimenta sucessos consecutivos realizando determinada tarefa, tornando-se confiante em seu desempenho para essa atividade específica. Uma vez estabelecida e aumentada, a $\mathrm{AE}$ tende a se generalizar para ouras situações semelhantes àquelas em que foi restaurada. As capacidades comportamentais também podem ser restauradas através da experiência vicária. Ver os outros desempenharem atividades consideradas ameaçadoras sem conseqüências adversas pode gerar expectativas nos observadores de que eles também melhorarão se intensificarem e persistirem em seus esforços. Dessa maneira, quando são mostrados os ganhos alcançados pelo comportamento de enfrentamento com esforço, minimiza para os observadores o impacto negativo da 
angústia temporária e demonstra que mesmo o mais ansioso pode ser bem-sucedido pela perseverança.

Outra fonte descrita pelo autor é a persuasão verbal, isto é, mediante sugestões as pessoas são levadas a acreditar que podem enfrentar com sucesso o que as derrotou no passado. As experiências induzidas são mais fracas do que as de sucesso direto. Os estados fisiológicos são outra fonte de informação apontada pelo autor. Esses estados também informam sobre as capacidades do indivíduo, uma vez que, dependendo de como ele se sente diante de uma determinada situação, fará um julgamento de suas habilidades. A alta excitação, usualmente, debilita o desempenho e, sendo assim, os indivíduos provavelmente esperam mais sucesso quando não estão assediados por excitações aversivas do que quando estão tensos e visceralmente agitados.

As autocrenças das pessoas em suas capacidades permitem que elas exerçam controle sobre os eventos que afetam suas vidas e sobre como essas crenças se traduzem em realização e motivação. As crenças de $\mathrm{AE}$ são elementos-chave da agência humana, uma vez que são fortes preditores do nível de realizações que os indivíduos atingem e têm forte impacto no pensamento, no afeto, na motivação e na ação (Berry \& West, 1993).

As crenças de AE moldam os resultados que as pessoas esperam que seus esforços produzam. Aqueles com alta AE esperam alcançar resultados favoráveis. As crenças de AE também determinam como os obstáculos e os impedimentos são vistos. Pessoas com baixa AE são facilmente convencidas da futilidade de seus esforços diante das dificuldades e rapidamente desistem de continuar tentando. Aqueles indivíduos com alta AE vêem os impedimentos como transponíveis pelo aperfeiçoamento das habilidades de autogerenciamento e pelos esforços perseverantes. Estes permanecem no curso de suas ações diante de dificuldades (Bandura, 1997).

O controle comportamental permite ao indivíduo lidar com aspectos aversivos e afeta a forma como o ambiente é percebido por ele. Situações de estresse que podem ser controladas são interpretadas como menos ameaçadoras, e essa avaliação cognitiva reduz a excitação antecipatória e dirige a motivação para a ação. O impacto da informação na expectativa de eficácia irá depender de como essa informação é avaliada cognitivamente. As circunstâncias sociais, situacionais e temporais sobre a ocorrência dos eventos entrarão em tal avaliação (Bandura, 1977).

\section{Auto-eficácia percebida e o manejo de condições crônicas e incapacidades}

De acordo com Toshima, Kaplan e Ries (1992), o conceito de AE tem recebido substancial suporte Psico-USF, v. 12, n. 1, p. 75-81, jan./jun. 2007 empírico sobre seu papel explanatório no processo de mudança de hábitos de saúde, na manutenção do tratamento prescrito e no manejo das conseqüências das doenças crônicas. O senso de eficácia pessoal não apenas facilita o autogerenciamento de desordens físicas, mas também diminui seus efeitos emocionais. Quando afetados pela severidade e pela cronicidade das doenças, os indivíduos com alta eficácia percebida são menos eestresseados e menos deprimidos pelas suas condições e usam melhores estratégias de enfrentamento do que aqueles com baixa AE (Bandura, 2004).

A AE percebida influencia o que as pessoas escolhem fazer, sua motivação, sua perseverança diante das dificuldades, sua vulnerabilidade ao estresse e à depressão. Influenciam na situação de presença de doenças crônicas inclusive no nível de benefício que os indivíduos recebem das intervenções terapêuticas. Assim, a AE percebida provê um mecanismo de ligação entre fatores psicossociais e o status funcional. Altos níveis de AE para o enfrentamento das conseqüências das doenças crônicas estão associados com a melhora nos sintomas, o melhor bem-estar físico e emocional e $\mathrm{o}$ aumento das atividades sociais. Assim, a AE percebida é uma pré-condição essencial para o manejo apropriado da doença crônica (Holman \& Lorig, 1992).

Baixas expectativas de AE percebida podem predizer grande incapacidade e, para as pessoas idosas, centram-se principalmente na reavaliação e na avaliação incorreta de suas capacidades. De fato, muitas capacidades físicas podem declinar na velhice, especialmente diante de doenças, requerendo reavaliações da eficácia pessoal. A avaliação da própria capacidade é altamente vantajosa e freqüentemente é essencial para o funcionamento efetivo. Aqueles que julgam incorretamente o que são capazes de fazer podem engajar-se em desempenhos que produzem conseqüências pouco satisfatórias (Bandura, 1997).

Considerando que a doença crônica não pode ser curada, ela precisa ser manejada ao longo do tempo. Isso requer, entre outras coisas, melhora na dor, acentuação e manutenção do funcionamento com a incapacidade funcional crescente e o desenvolvimento de habilidades auto-regulatórias compensatórias (Bandura, 1997). O objetivo é retardar o progresso da incapacidade e melhorar a qualidade de vida das pessoas afetadas. Programas de autogerenciamento baseados no modelo de AE melhoram a qualidade da saúde e reduzem a necessidade de serviços médicos (Bandura, 2004). Segundo Lorig e cols. (2001), os resultados de um programa de autogerenciamento de doenças crônicas para pessoas acima de 40 anos mostrou uma redução significativa no distress, um aumento na eficácia percebida associada ao menor uso dos serviços de 
saúde. Apesar da incapacidade física ter piorado, os participantes mantiveram ou melhoraram todos os outros aspectos do seu status de saúde e suas atividades e papéis sociais não declinaram.

Muitos tipos de práticas de autogerenciamento são benéficas e exeqüíveis, e a $\mathrm{AE}$ percebida para executar estas práticas e para manejar as conseqüências da doença melhora os resultados de saúde grandemente. Autogerenciamento significa ter ou estar preparado para obter as habilidades e recursos necessários para melhor acomodar a doença crônica e suas conseqüências (Holman \& Lorig, 1992).

Segundo Lorig e cols. (2001), com relação às doenças crônicas, grandes avanços acontecem no cuidado médico e cirúrgico, mas pouco é feito para permitir ao paciente manejar sua doença ao longo do tempo. Os pacientes têm que enfrentar o desconforto e a incapacidade, seguir o tratamento regularmente, modificar comportamentos para minimizar os resultados indesejáveis, ajustar sua vida social e o trabalho às suas limitações funcionais e ainda lidar com as conseqüências emocionais.

\section{Auto-eficácia e incapacidade funcional: dados de pesquisa}

Imuta, Yasumura, Abe e Fukao (2001) examinaram a prevalência e as características psicossociais de idosos fragilizados no Japão. Os resultados sugerem que existe uma forte associação entre fatores psicossociais e fragilidade em idosos. Observaram que idosos fragilizados apresentaram AE mais baixa e pior saúde percebida do que idosos independentes. Kono, Kai, Sakato e Rubenstein (2004) investigaram a freqüência de sair e mover-se para fora de casa como um preditor de mudanças funcionais e psicossociais entre idosos fragilizados no Japão. Verificaram que os idosos que saíam mais freqüentemente eram menos incapacitados, mais socialmente ativos e menos depressivos do que aqueles que saíam com menos freqüência. Aqueles que saíam com mais freqüência melhoraram, mudando suas crenças de AE tanto para atividades diárias quanto para as atividades de promoção da saúde.

Seeman e Chen (2002) testaram em estudo longitudinal a hipótese de que estilo de vida (participação em atividades físicas regulares e engajamento social), fatores sociodemográficos e fatores psicológicos (crenças de $\mathrm{AE}$ e distress psicológico) influenciam no risco de declínio funcional ao longo do tempo em grupos de idosos com doença crônica (hipertensão, diabetes, doenças cardiovasculares, câncer e fraturas). De acordo com os resultados obtidos, os fatores psicossociais mostraram associações independentes e significantes nos padrões de mudança no funcionamento físico entre idosos com condições crônicas. Quando comparados com idosos sem condições crônicas, os sujeitos exibiram escores mais baixos em $\mathrm{AE}$ e maiores em distress psicológico, e pior funcionamento físico. Verificaram que, em especial para aqueles com histórico de câncer, menor declínio foi observado nos indivíduos com maior crença de $\mathrm{AE}$ em atividades instrumentais (habilidade percebida em lidar com tarefas relativas às finanças, transporte, situação de moradia e o senso de ser produtivo). Segundo os autores, para esses indivíduos, o impacto mais direto na funcionalidade é geralmente resultado de cirurgias e/ou quimioterapia e os fatores psicossociais podem impactar na extensão na qual os indivíduos trabalham para recuperar e/ou manter altos níveis de funcionalidade.

Kempen e cols. (1999) investigaram em que medida três recursos psicológicos (neuroticismo, domínio e expectativa de AE) moderam os efeitos da incapacidade em idosos residentes na comunidade. Verificaram que os atributos psicológicos influenciam na incapacidade, mas este efeito não é moderador. $\mathrm{O}$ neuroticismo e o senso de domínio influenciam mais fortemente o desempenho de papéis sociais, e as expectativas de AE, as atividades básicas e instrumentais de vida diária.

Jang, Haley, Small e Motimer (2002) buscaram avaliar o potencial dos fatores psicológicos (senso de domínio, conceito relacionado à $\mathrm{AE}$ ) e dos fatores sociais (rede social, suporte social e satisfação com o suporte) em alterar a associação entre a incapacidade e a depressão em idosos residentes na comunidade. Os resultados indicaram que alto senso de domínio e grande satisfação com o suporte amenizam o impacto da incapacidade na depressão, isto é, há menor chance de experimentar sintomas depressivos na presença de incapacidades. Verificaram que idosos com grande nível de incapacidade tendem a ter menor senso de domínio. Grandes níveis de depressão foram observados nos idosos mais velhos, com mais condições crônicas, com maior incapacidade e menor senso de domínio. O senso de domínio permite aos indivíduos prevenir ou manejar efetivamente os problemas de saúde, ajuda na mobilização efetiva dos recursos pessoais e estratégias de enfrentamento e pode alterar as conseqüências negativas da incapacidade, facilitando o ajustamento.

De acordo com Grace e cols. (2002), a AE é um preditor da recuperação, do manejo e do funcionamento físico, psicológico e social do indivíduo. Altos níveis de $\mathrm{AE}$ estão associados com baixos níveis de distress psicológico e em indivíduos com doenças crônicas têm efeitos favoráveis e diretos nos sintomas 
depressivos. Para os autores, no contexto das doenças cardiovasculares, as mulheres estão particularmente em risco psicossocial, uma vez que apresentam maior sintomatologia depressiva e ansiógena, menos suporte social e menor AE, fato que diminui as taxas de aderência a programas de reabilitação por parte das mesmas.

Para Denison e Lindberg (2004), a AE influencia no ajustamento à condição de dor e da incapacidade associada e medeia a relação entre intensidade da dor, incapacidade e depressão em pacientes com dor crônica. A AE pode explicar por que os pacientes persistem em confrontar as atividades diárias diante de obstáculos como a dor. Os autores verificaram, em sua pesquisa com pacientes com dor musculoesquelética em cuidado primário, que as crenças de AE são determinantes mais importantes da incapacidade do que a evitação, a intensidade e a duração da dor.

Para Arnstein, Caudill, Mandle, Norris e Beasley (1999), o nível de AE contribui significativamente na extensão na qual a pessoa está incapacitada por sua dor crônica. Os autores investigaram as crenças de $\mathrm{AE}$ como mediadoras da incapacidade associada à dor em pacientes com dor crônica. Os resultados mostram que a $\mathrm{AE}$ medeia a relação entre intensidade da dor e a incapacidade associada. Isso indica a possibilidade de que indivíduos com dor crônica podem tornar-se incapacitados, em parte em virtude das baixas crenças de AE. Ou seja, a dúvida sobre a própria capacidade, mesmo se a dor é leve ou moderada em intensidade, pode ser incapacitante. Assim, a terapia deveria perseguir múltiplas metas, incluindo a redução da dor, a melhora da funcionalidade e o aumento das crenças de AE.

De acordo com Kaplan, Wurtele e Gillis (1996), as expectativas de $\mathrm{AE}$ influenciam no nível de esforço que o indivíduo com dor crônica irá despender em várias atividades, inclusive durante testes de avaliação da capacidade funcional desses pacientes. Para os autores, nem todos os pacientes dão o máximo de esforço durante essas avaliações, assim, existe o risco do avaliador tomar decisões equivocadas quanto às capacidades dos pacientes. Por causa da importância de se tomar decisões corretas, os autores procuraram examinar a relação entre fatores psicológicos (AE, incapacidade auto-relatada, ansiedade e depressão) e o máximo de esforço despendido durante avaliação da capacidade funcional em pacientes com dor lombar. Verificaram que os pacientes que se percebiam com menor AE, mais depressão e mais ansiosos estão em risco de não dar o máximo de esforço durante a avaliação. Sendo assim, os profissionais precisam Psico-USF, v. 12, n. 1, p. 75-81, jan./jun. 2007 considerar como os fatores psicológicos podem afetar o desempenho do paciente durante a avaliação da capacidade funcional e facilitar o preparo psicológico adicionalmente ao físico.

Segundo Keefe e cols. (2002), a AE está emergindo como um dos fatores mais importantes no entendimento da dor e da incapacidade em pessoas com artrite. Tanto estudos longitudinais quanto transversais mostram que a AE é importante na predição dos índices de dor e incapacidade e que atua como mediadora da dor e do status psicológico em pessoas que sofrem de artrite. As pessoas que vivem com artrite variam substancialmente em sua AE. Algumas com alto nível de $\mathrm{AE}$ são confiantes em relação as suas habilidades de enfrentamento da dor e outros sintomas, enquanto outras, com baixo senso de eficácia pessoal, sentem-se incapazes de manejar sua doença. Assim, para os autores, mediante a consistência das evidências relativas à importância das crenças de eficácia percebida, os educadores de saúde estão cada vez mais incorporando em seus programas educacionais intervenções com o objetivo de aumentar o senso de AE.

Barry, Guo, Kerns, Duong e Reid (2003) examinaram a relação entre $\mathrm{AE}$ funcional (nível de confiança em sua própria habilidade em desempenhar cada uma de dez atividades requeridas para independência funcional) e a incapacidade decorrente da dor em homens idosos com dor crônica. Verificaram que a $\mathrm{AE}$ funcional é um fator forte e independente associado com a incapacidade relacionada à dor, isto é, na medida em que o nível de AE funcional decresce, a probabilidade da incapacidade aumenta. Os resultados corroboram a importância de intervenções que visem modificar as crenças de eficácia percebida.

Os estudos apresentados indicam que as crenças de AE influenciam nos índices de incapacidade e podem atuar como mediadoras do status psicológico de idosos afetados por condições crônicas. Foi possível perceber que quanto mais crenças de eficácia pessoal, menor a incapacidade funcional, mais saúde percebida, menos distress psicológico, menor o declínio em atividades básicas e instrumentais de vida diária, menos sintomas depressivos, mais ajustamento à dor, maior o esforço despendido em atividades requeridas e mais ajustamento pessoal e à capacidade de mobilização de recursos pessoais de enfrentamento.

As crenças de eficácia pessoal funcionam como um forte elemento contra os efeitos adversos da incapacidade, porque influenciam na motivação para o enfrentamento dos estressores e no manejo do ambiente social e físico. Uma vez dotadas de habilidades e crenças em suas capacidades, as pessoas são mais capazes de adotar comportamentos que 
promovem a saúde, de eliminar aqueles que a incapacitam e de obter melhores resultados nos tratamentos de incapacidades físicas (Bandura, 2004).

\section{Considerações finais}

Pode-se dizer que o controle e o exercício das atividades diárias na presença de doenças crônicas e incapacidades são parcialmente mediados pelas variações das crenças de eficácia. Aquele com maior senso de AE busca papéis ativos em relação ao manejo de sua condição de saúde. Segundo Bandura (2004), as limitações funcionais são mais governadas pelas crenças na própria capacidade do que pelo nível atual da incapacidade física. Para o autor, reavaliações cognitivas positivas que focam nos aspectos da própria vida que são pessoalmente controláveis podem aumentar a eficácia percebida, o que ativa muitos processos adaptativos no enfrentamento de condições crônicas de saúde.

A maior incidência de eventos negativos na velhice, como doenças, perdas e acidentes, levanta a questão de como os idosos lidam de maneira bemsucedida com eles e conseguem manter uma boa qualidade de vida, ou pelo menos a melhor possível. A deficiência na competência comportamental afeta a vida em geral dos idosos, seu bem-estar e a necessidade de ajuda informal e serviços de saúde (Melzer \& Parahyba, 2004). Nesse sentido, torna-se importante a compreensão dos caminhos pelos quais os idosos enfrentam suas perdas em funções físicas e papéis sociais e a extensão na qual os recursos psicológicos e sociais operam sobre os efeitos negativos das condições crônicas e incapacitantes. No estudo do envelhecimento, um aspecto crítico é verificar como o idoso mantém e exercita o seu senso de AE, o que não é fácil em razão das transições da vida, das mudanças biopsicológicas e das barreiras sociais com as quais eles se deparam (Bandura, 1997).

\section{Referências}

Agewall, S., Wikstrand, J., Dahlöf, C. \& Fagerberg, B. (1996). Negative feelings (discontent) predict progress of intima-media thickness of the commom carotid artery in treated hypertensive men at high cardiovascular risk. American Journal of Hypertension, $9(6), 545-550$.

Arnstein, P., Caudill, M., Mandle, C. L., Norris, A. \& Beasley, R. (1999). Self-efficacy as the mediator of the relationship between pain intensity, disability and depression in chronic pain patients. Pain, 80(3), 483491.

Bandura, A. (1977). Self-efficacy: toward a unifying theory of behavioral change. Psychological Review, 84(2), 191-215.

Bandura, A. (1997). Self-efficacy: the exercise of control. New York: W. H. Freeman and Company.

Bandura, A. (2004). Health promotion by social cognitive means. Health Education \& Behavior, 31(2), 143-164.

Barry, L. C., Guo, Z., Kerns, R. D., Duong, B. D. \& Reid, M. C. (2003). Functional self-efficacy and painrelated disability among older veterans with chronic pain in a primary care setting. Pain, 104(1-2), 131137.

Berry, J. M. \& West, R. L. (1993). Cognitive self-efficacy in relation to personal mastery and goal setting across the life span. International Journal of Behavioral Development, 16, 351-379.

Denison, E. \& Lindberg, P. (2004). Self-efficacy, fear avoidance and pain intensity. Pain, 111(3), 245-252.

Grace, S. L., Abbey, S. E., Shnek, Z. M., Irvine, J., Franche, R. L. \& Stewart, D. E. (2002). Cardiac rehabilitation. Review of Psychosocial Factors. General Hospital Psychiatry, 24(3), 121-126.

Holman, H. \& Lorig, K. (1992). Perceived self-efficacy in self-management of chronic disease. Em Schwarzer, R. Self-efficacy: Thought control and action (pp. 305-323). London: Taylor \& Francis.

Imuta, H., Yasumura, S., Abe, H. \& Fukao, A. (2001). Prevalence and psychosocial characteristics of the frail elderly in Japan: A community-based study. Aging: Clinical and Experimental Research, 13(6), 443453.

Jang, Y., Haley, W. E., Small. B. J. \& Motimer, J. (2002). The role of mastery and social resources in the associations between disability and depression in later life. The Gerontologist, 42(6), 807-813.

Jette, A. M. (1996). Disability trends and transitions. Em Binstock, R. H. \& George, L. K. Handbook of aging and the social science (pp. 94-116). San Diego: Academic Press.

Kaplan, G. M., Wurtele, S. K. \& Gillis, D. (1996). Maximal effort during functional capacity evaluations: An examination on psychological factors. Arch. Phys. Med. Rehabil., 77, 161-164.

Keefe, F. J., Smith, S. J., Buffington, A. L. H., Gibson, J., Studts, J. L. \& Caldwell, D. S. (2002). Recent 
advances and future directions in the biopsychosocial assessment and treatment of arthritis. Journal of Consulting and Clinical Psychology, 70(3), 640-655.

Kempen, G. I. J. M., Van Heuvelen, M. J. G., Van Sonderen, E., Van Denbrink, R. H. S., Kooijman, A. C. \& Ormel, J. (1999). The relationship of functional limitations to disability and the moderating effects of psychological attributes in community dwelling older persons. Social Science \& Medicine, 48(9), 1161-1172.

Kono, A., Kai, I., Sakato, C. \& Rubenstein, L. Z. (2004). Frequency of going outdoors: A predictor of functional and psychosocial change among ambulatory frail elders living at home. Gerontological Society of America, 59(3), 275-280.

Lamb, V. L. (1996). A cross-national study of quality of life factors associated with patterns of elderly disablement. Social Science and Medicine, 42(3), 363-377.

Lima-Costa, M. F., Barreto, S. M. \& Giatti, L. (2003). Condições de saúde, capacidade funcional, uso de serviços de saúde e gastos com medicamentos da população idosa brasileira: um estudo descritivo baseado na Pesquisa Nacional por Amostra de Domicílios. Cadernos de Saúde Pública, 19(3), 735-743.

Lorig, K., Ritter, P., Stewart, A. L., Sobel, D. S., Brown, B. W., Bandura, A., Gonzalez, V. M., Laurent, D. D. \& Holman, H. R. (2001). Chronic disease selfmanagement program: 2-year health status and health care utilization outcomes. Medical Care, 39(11), 12171223.

Melzer, D. \& Parahyba, M. I. (2004). Sociodemografic correlates of mobility disability in older brazilians: Results of the first national survey. Age and Ageing, 33(3), 253-257.

Seeman, T. \& Chen, X. (2002). Risk and protective factors for physical functioning in older adults with and without chronic conditions: Mac Arthur Studies of Successful Aging. Journal of Gerontology: Series B: Psychological Sciences and Social Sciences, 57(3), 135-144.

Smith, J., Borchelt, M., Maier, H. \& Jopp, D. (2002). Health and well-being in the young and oldest old. Journal of Social Issues, 58(4), 715-732.

Toshima, M. T., Kaplan, R. M. \& Ries, A. L. (1992). Self-efficacy expectancies in chronic obstructive pulmonary disease rehabilitation. Em R. Schwarzer. Self-efficacy: thought control and action (pp. 325-354). London: Taylor \& Francis.

Xavier, F. M. F., Ferraz, M. P. T., Marc, N., Escostequy, N. U. \& Moriguchi, E. H. (2003). Elderly people's definition of quality of life. Revista Brasileira de Psiquiatria, 25(1), 310-317.

Recebido em novembro de 2005 Reformulado em novembro de 2006 Aprovado em fevereiro de 2007

Sobre as autoras:

Dóris Firmino Rabelo é psicóloga graduada pela Universidade Federal de Uberlândia - UFU e mestre em Gerontologia pela Universidade Estadual de Campinas - Unicamp. Atualmente é coordenadora do Núcleo de Proteção Social do Idoso da Prefeitura Municipal de Uberlândia (MG) e coordenadora do curso de Especialização em Gerontologia da UNIMINAS (Uberlândia, MG).

Chrystiane Mendonça Cardoso é psicóloga graduada pela Universidade Católica de Goiás - UCG e mestre em Psicologia Aplicada (área temática - Psicologia da Saúde) pela Universidade Federal de Uberlândia - UFU. Atualmente desenvolve trabalho de Psicologia da Saúde junto à Prefeitura Municipal de Tupaciguara (MG), atuando diretamente nos programas de educação para a saúde com pacientes com doenças crônicas (hipertensão e diabetes). 
